\title{
Caracterización en niños de 4 a 6 años con interferencias dentarias de círculos infantiles
}

\author{
Characterization in children aged 4 to 6 years with dental interference from children's daycare \\ institutions
}

\author{
Bárbara Olaydis Hechavarría Martínez¹, Leonardo Núñez Antúnez ${ }^{1}$ \\ Yisel Pérez Pérez ${ }^{1}$, Leanne Bárbara Pons Hechavarría ${ }^{4}$, Nayra Ivonne Núñez Almarales ${ }^{5}$
}

\section{RESUMEN}

Objetivo: Caracterizar a los niños de 4 a 6 años de edad de círculos infantiles del área de salud del Policlínico Docente Julián Grimau García de Santiago de Cuba, según variables clínicas y epidemiológicas. Materiales y método: estudio observacional, descriptivo y transversal en niños de 4 a 6 años de edad pertenecientes a los círculos infantiles del área de salud del Policlínico Docente Julián Grimau García de Santiago de Cuba, de Julio de 2016 a Abril de 2017. La muestra estuvo constituida por 57 niños de 4 a 6 años de edad de ambos sexos, pertenecientes a los 2 círculos infantiles del área de salud anteriormente indicada y que presentaron interferencias dentarias. Se realizó una entrevista estructurada a padres o tutores, con el fin de obtener datos precisos y verificados, utilizando como medida de resumen el porcentaje. Se realizó el examen bucal a los niños utilizando el depresor lingual y luz natural o artificial. Resultados: Predominó el sexo masculino y el periodo de dentición temporal. La respiración bucal y el uso del biberón fueron los hábitos deformantes más practicados. Conclusiones: la mayoría de niños examinados no presentaron anomalías de oclusión. Se recomienda realizar estudios analíticos sobre interferencias dentarias en niños como factor causal de maloclusiones, elevar las actividades de promoción y prevención de salud a las embarazadas y madres sobre la importancia de practicar estilos de vida favorables para la salud y el desarrollo del sistema estomatognático de sus hijos desde edades tempranas.

PALABRAS CLAVES: Hábitos deformantes; Niños; Maloclusiones; Estilos de vida; Promoción.

\begin{abstract}
Objective: the objective of characterizing children from 4 to 6 years of age from nursery schools in the health area of the "Julián Grimau García" Teaching Polyclinic in Santiago de Cuba, according to clinical and epidemiological variables. Materials and methods: An observational, descriptive and cross-sectional study was carried out in children from 4 to 6 years of age belonging to the nursery circles of the health area of the "Julián Grimau García" Teaching Polyclinic in Santiago de Cuba, in the period of July 2016 to April 2017 .The study was constituted by the 57 children from 4 to 6 years of age belonging to the 2 children's circles of the aforementioned health area, of both sexes and who presented dental interferences. To obtain the data, a structured interview was conducted with parents or guardians, in order to obtain accurate and verified data, using the percentage as a summary measure. The oral examination was performed on children using the tongue depressor and natural or artificial light. Results: the male sex and the period of temporary dentition predominated. Buccal breathing and bottle use were the most practiced deforming habits. Conclusions: Concluding that most of the children examined did not present anomalies of the occlusion. Considering this, we recommend carrying out analytical studies in the area that allow deepening the study of dental interferences in children as a causal factor of malocclusions, elevating health promotion and prevention activities to pregnant women and mothers on the importance of practicing favorable lifestyles for the health and development of the stomatognathic system of their children from an early age.
\end{abstract}

KEYWORDS: Deforming habits; Children; Malocclusions; Lifestyles; Promotion

${ }^{1}$ Universidad de Ciencias Médicas de Santiago de Cuba, Facultad de Estomatología,

Departamento de Estomatología General Integral. Santiago de Cuba, Cuba.

Correspondencia:

Dra. Bárbara Olaydis Hechavarría Martínez

Facultad de Estomatología, Santiago de Cuba, Cuba.

Correo electrónico: barbaraolaidis@infomed.sld.cu
Este es un artículo Open Access distribuido bajo la la licencia Creative Commons Atribución-No comercial -Compartir igual

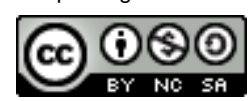


Citar como:. Hechavarria B, Nuñez L, Perez Y, Pons L, Nuñez N. Caracterización en niños de 4 a 6 años con interferencias dentarias de círculos infantiles. KIRU. 2020;17(2): 75- 83. https://doi.org/10.24265/kiru.2020.v17n2.03

\section{INTRODUCCIÓN}

En la boca se encuentran una serie de estructuras anatómicas donde convergen importantes funciones de comunicación con el exterior: respiración, fonación, gustación y sobre todo, masticación. Es el aparato masticatorio el campo de interés estomatológico y hacia él se dirige la atención de todas las especialidades derivadas de la estomatología (1).

El estudio y conocimiento detallado de la oclusión dentaria ha sido a través de los años indispensable para la estomatología, llegando a ser una de las temáticas más controvertidas. Algunos autores la han definido como el conjunto de relaciones que se establecen como resultado de los contactos oclusales entre los elementos integrantes del sistema estomatognático ${ }^{(2)}$.

El concepto moderno de oclusión incluye la idea de un sistema estructurado e integrado de unidades funcionales que abarcan dientes, articulaciones, músculos de la masticación y del cuello; también debe referirse al estudio de las alteraciones que se constituyen por mal función del sistema, cuando se altera o falla uno o más componentes del mismo; al estudio de la dinámica mandibular o al estudio de las relaciones dinámicas del sistema estomatognático ${ }^{(3)}$.

La armoniosa correlación entre estos componentes del aparato masticatorio es de primordial importancia para la capacidad funcional y el mantenimiento de dicho aparato ${ }^{(4)}$. La oclusión buena o mala es el resultado de una síntesis intrincada y complicada de relaciones genéticas y ambientales que actúan durante las etapas tempranas del desarrollo de la niñez y el principio de

la edad adulta. Los aspectos genéticos de la oclusión se relacionan con los patrones del crecimiento del maxilar y la mandíbula (5).

Una interferencia oclusal es cualquier contacto dentario que evita que las demás superficies oclusales logren contactos estables y armoniosos, las mismas que impiden o interfieren en la armonía de los movimientos mandibulares excéntricos ${ }^{(6)}$.

Los factores ambientales desempeñan un papel principal en el desarrollo dental y en la formación de la oclusión dental. Estos factores incluyen "fuerzas" que posicionan a los dientes en la boca cuando hacen erupción y que conservan un equilibrio una vez que han erupcionado y establecido la oclusión ${ }^{(7,8)}$.
Las correctas relaciones oclusales son la base del adecuado funcionamiento del aparato estomatognático y, en gran medida, de la estética del rostro. Cuando están alteradas, pueden aparecer trastornos oclusales e incluso hábitos perniciosos, por lo que una oclusión funcional significa un estado de oclusión dentaria en el cual las superficies oclusales no presentan obstáculos o interferencias para los movimientos suaves de deslizamiento de la mandíbula ${ }^{(9,10)}$.

Según la Organización Mundial de la Salud, las maloclusiones ocupan el tercer lugar en prevalencia entre las afecciones bucodentales, luego de las caries y la enfermedad periodontal (9). En Latinoamérica, según datos de la Organización Panamericana de la Salud, las interferencias dentarias afectan a más de $80 \%$ de las poblaciones y constituyen uno de los motivos de consulta más frecuentes en las clínicas estomatológicas ${ }^{(11)}$.

Las interferencias oclusales que constituyen un problema habitual en las denticiones temporal y mixta se asocian a maloclusiones funcionales como mordidas cruzadas anteriores y posteriores, anomalías faciales, mordidas abiertas anteriores, desviaciones de la línea media y afectaciones de la articulación temporomandibular, puesto que obstaculizan las relaciones que se establecen entre los arcos dentarios impidiendo el trayecto armonioso de la mandíbula desde la posición postural hasta los movimientos de la dinámica mandibular, los que no tratados a tiempo pueden provocar alteraciones esqueletales ${ }^{(6)}$.

En encuestas epidemiológicas del Programa de Atención al menor de 19 años del área de Salud de la Clínica Estomatológica Provincial Docente de Santiago de Cuba, se obtuvo que en 705 niños de 6 años de edad, el $52 \%$ presentó interferencias dentarias ${ }^{(12)}$.

Según perfil epidemiológico realizado en consultas de Ortodoncia Interceptiva en el área de salud del Policlínico Docente Julián Grimau García de Santiago de Cuba, aproximadamente el $30 \%$ de los niños que cursan el quinto año de vida y pre-escolar de las instituciones infantiles presentaban interferencias dentarias.

Si las interferencias no son detectadas y corregidas tempranamente, las anomalías que se produzcan pueden demandar tratamientos ortodóncicos con aparatos fijos que son más costosos e incluso podrían hasta requerir de los servicios de Cirugía Ortognática, con afectación funcional y estética del paciente. 
Por lo antes expuesto y teniendo en cuenta que no existen investigaciones previas sobre el tema en nuestra área de salud, surgió la motivación de realizar esta investigación, lo cual contribuirá a lograr un beneficio socioeconómico, puesto que sus resultados permitirán implementar medidas de promoción y prevención de salud adecuadas.

El objetivo del estudio fue caracterizar a los niños de 4 a 6 años de edad con interferencias dentarias de los Círculos Infantiles del área de salud del Policlínico Docente Julián Grimau García de Santiago de Cuba, según variables clínicas y epidemiológicas.

\section{MATERIALES Y MÉTODOS}

La investigación se llevó a cabo teniendo en cuenta consideraciones éticas relacionadas con los pacientes sujetos de estudio, para lo cual se siguieron las normas planteadas en la declaración de Helsinki y previa autorización de la Dirección Municipal de Educación y de los directores de los círculos infantiles pertenecientes al área de salud del Policlínico Docente Julián Grimau García de Santiago de Cuba. Se solicitó la aprobación de padres y/o tutores de los pacientes por ser menores de edad, previa explicación e información del contenido del trabajo. Para completar la ficha de vaciamiento de datos, se procedió a realizar un examen clínico intrabucal y funcional en los departamentos de enfermería de las propias escuelas y otros locales acondicionados para ello. Con la participación de 2 estomatólogos y 2 estudiantes del quinto año de la carrera Estomatología, además de las enfermeras de dichos departamentos.

Se realizó un estudio transversal en niños de 4 a 6 años de edad pertenecientes a los círculos infantiles del área de salud del Policlínico Docente Julián Grimau García de Santiago de Cuba, en el período comprendido de julio del 2016 a abril del 2017, con el objetivo de caracterizar a los pacientes con interferencias dentarias según variables clínicas y epidemiológicas.

El universo de estudio estuvo constituido por los 57 niños de 4 a 6 años de edad pertenecientes a los 2 círculos infantiles del área de salud anteriormente citada, de ambos sexos y que presentaron interferencias dentarias.

Para dar salida al objetivo propuesto se tuvieron en cuenta las siguientes variables.

1. Edad: Variable cuantitativa continúa. Se tomó la edad cumplida en años al momento del estudio.

2. Sexo: Variable cualitativa nominal dicotómica. Se consideraron las dos categorías biológicas:
- Femenino

- Masculino

3. Lactancia: Variable cualitativa nominal dicotómica.

Definición: Nutrición a base del consumo de leche y forma de administrarse desde el momento del nacimiento. Existen dos tipos de lactancia:

Lactancia materna: Cuando fue exclusiva y se realizó hasta los 6 meses.

Lactancia mixta: Cuando se incorporó antes de los 6 meses otros tipos de alimentos con leche.

Escala:

a. Lactancia adecuada: Cuando lactancia materna fue exclusiva hasta los 6 meses.

b. Lactancia no adecuada: Cuando no fue exclusiva la lactancia hasta los 6 meses.

4. Dieta: Variable cualitativa nominal dicotómica. Definición: Conjunto de productos (líquidos y sólidos) que se consumen de forma habitual, siendo esta:

- Dieta blanda: Aquella dieta en que los alimentos se ingieren blandos tipo puré, ya sea porque se usó la batidora 0 se aplastaron.

- Dieta sólida: Cuando se consumen los alimentos en su forma natural o cocidos, dieta de la familia.

Escala:

a. Dieta adecuada: Cuando se efectúo la dieta sólida a partir del primer año de vida.

b. Dieta no adecuada: Cuando continuó la dieta blanda a partir del primer año de vida.

\section{Hábitos deformantes.}

a. Hábito de succión digital: Variable cualitativa nominal dicotómica

Definición: Patrón neuromuscular de naturaleza compleja y consiste en introducir 1 o más dedos en la boca habitualmente.

Escala:

- Sí: Cuando el niño se introdujo 1 o más dedos en la boca.

- No: Cuando sea lo contrario a la descripción anterior.

b. Hábito postural: Variable cualitativa nominal dicotómica

Definición: cuando el niño adopta posiciones inadecuadas que generan presiones anormales sobre los maxilares y los dientes al dormir 0 al sentarse.

Escala: 
- Sí: Cuando el niño adoptó posiciones inadecuadas, al dormir, sentarse, en busca de apoyo con la mano contra la cara.

- No: Cuando sea lo contrario a la descripción anterior.

c. Hábito uso del biberón: Variable cualitativa nominal dicotómica.

Definición: Cuando el niño ingiere tomas de leche con el biberón.

Escala:

- Sí: Cuando el niño presentó este estilo de alimentación

- No: Cuando sea lo contrario a la descripción anterior.

6. Función Respiratoria: Variable cualitativa nominal dicotómica.

Definición: Es una función orofaríngea vital en la que el ser humano toma el aire y esta puede realizarse por la cavidad bucal o nasal.

- Respiración bucal: Se diagnosticó cuando el paciente realizó esta función por la cavidad bucal en la respiración relajada.

- Respiración nasal: Se diagnosticó cuando el paciente mantuvo los labios tocándose ligeramente durante la respiración relajada, realizándola por la cavidad nasal.

Escala:

- Disfunción respiratoria: Cuando coincidió con la respiración bucal.

- Función respiratoria normal: Cuando coincidió con la respiración nasal.

7. Tipo de dentición: Variable cualitativa nominal Definición: Conjunto de dientes con características morfofuncionales definidas para los cambios que se van produciendo durante el desarrollo de la oclusión.

- Dentición temporal: Es la primera dentición que aparece en la cavidad en el niño.

- Dentición mixta temprana: Conjunto de dientes permanentes que aparecen con el brote del primer molar permanente y termina con la caída de los caninos temporales inferiores.

Escala:

- Temporal: Cuando estuvieron presentes solamente dientes temporales.

- Mixta temprana: Cuando estuvo presente algún molar permanente $\mathrm{y} / \mathrm{o}$ diente permanente del sector anterior.
8. Presencia de Anomalías: Variable cualitativa nominal dicotómica.

Definición: Cuando en la oclusión con interferencias dentarias se pudieron establecer las siguientes relaciones:

- Mordida abierta anterior: Se diagnosticó cuando los dientes anteriores no presentaron relación de oclusión localizada en sentido vertical, mientras los dientes remanentes estuvieron en oclusión.

- Seudo clase III funcional: Se diagnosticó cuando estuvieron presentes dientes del sector anterior, cuyas caras vestibulares de los incisivos superiores estuvieron por detrás de las caras linguales de los incisivos inferiores en el momento de cierre.

- Oclusión invertida posterior unilateral: Se diagnosticó cuando las cúspides vestibulares de los dientes posterosuperiores ocluyeron por lingual de las cúspides vestibulares de los dientes inferiores o borde a borde en un lado de la mandíbula.

Escala:

- Sin anomalías: Cuando en posición de relación céntrica no se observaron ninguna de las anomalías anteriores.

- Con anomalías: Cuando en posición de relación céntrica se observaron algunas de las anomalías anteriores.

Para lograr un óptimo grado de validez científica, se llevó a cabo una revisión bibliográfica acerca del tema de este trabajo en diferentes unidades como la Biblioteca Médica Provincial, Biblioteca de la Clínica Estomatológica Provincial Docente y a través del MEDLINE, LILACS e Internet.

Los datos se obtuvieron mediante la visita a los círculos infantiles pertenecientes al área de salud núm. 12, Policlínico Docente Julián Grimau García de Santiago de Cuba, donde se realizó la guía de entrevista estructurada a padres y/o tutores, con el fin de obtener datos precisos y verificados, con previa autorización de la Dirección Municipal de Educación y los respectivos directivos de los círculos infantiles. Para completar la ficha de vaciamiento de datos, se procedió a realizar el examen clínico intrabucal y funcional en el departamento de enfermería de los propios círculos infantiles, mediante el uso de la luz artificial y con el auxilio en muchas ocasiones de depresores linguales. 
Toda la información fue procesada en una computadora Pentium V, con ayuda del sistema estadístico SPSS. La información primaria se plasmó en una hoja de cálculo de Microsoft Excel, con el fin de elaborar las tablas y los gráficos estadísticos que se utilizaron para la presentación de los resultados. Las medidas de resumen empleadas fueron las frecuencias absolutas y relativas.
En la tabla 1, se muestra la distribución del universo estudiado según edad y sexo, donde predominó el sexo masculino por encima del sexo femenino. Encontrándose el mayor número de niños en la edad de 5 años con 28 niños de ambos sexos representando el $49,2 \%$.

\section{RESULTADOS}

Tabla 1. Pacientes con interferencias dentarias de los Círculos Infantiles del área de salud del Policlínico Julián Grimau según edad y sexo

\begin{tabular}{|c|c|c|c|c|c|c|}
\hline \multirow{2}{*}{ Edad } & \multicolumn{2}{|c|}{ Femenino } & \multicolumn{2}{c|}{ Masculino } & \multicolumn{2}{c|}{ Total } \\
\cline { 2 - 7 } & No. & $\%^{*}$ & No. & $\%^{*}$ & No. & $\%^{*}$ \\
\hline $\mathbf{4}$ & 8 & 14,0 & 17 & 29,8 & 25 & 43,8 \\
\hline $\mathbf{5}$ & 12 & 21,1 & 16 & 28,1 & 28 & 49,2 \\
\hline $\mathbf{6}$ & 2 & 3,5 & 2 & 3,5 & 4 & 7,0 \\
\hline Total & 22 & 38,6 & 35 & 61,4 & 57 & 100 \\
\hline
\end{tabular}

* \% calculado en base al total de niños.

En la tabla 2, se muestra que ligeramente más de la mitad de los niños tuvieron una lactancia materna no adecuada, 29 para un 50,9\%, a expensas del sexo masculino que tuvo mayor representación en el estudio realizado.

Tabla 2. Niños de 4 a 6 años de edad con interferencias dentarias según lactancia y sexo

\begin{tabular}{|c|c|c|c|c|c|c|}
\hline \multirow{2}{*}{ Lactancia } & \multicolumn{4}{|c|}{ Sexo } & \multicolumn{2}{c|}{ Total } \\
\cline { 2 - 5 } & \multicolumn{2}{|c|}{ Femenino } & \multicolumn{2}{|c|}{ Masculino } & \multicolumn{2}{|c|}{} \\
\cline { 2 - 6 } & No & $\%^{*}$ & No & $\%^{*}$ & No & $\%^{*}$ \\
\hline Adecuada & 11 & 19,3 & 17 & 29,8 & 28 & 49,1 \\
\hline No adecuada & 11 & 19,3 & 18 & 31,6 & 29 & 50,9 \\
\hline Total & 22 & 38,6 & 35 & 61,4 & 57 & 100 \\
\hline * \% calculado en base al total de niños.
\end{tabular}

En la tabla 3, se relacionan la presencia de hábitos deformantes con el sexo, observándose que la respiración bucal fue el hábito que más predominó, presente en 23 niños, principalmente del sexo masculino, seguido del uso del biberón, fundamentalmente en el sexo femenino.

Tabla 3. Niños de 4 a 6 años de edad con interferencias dentarias según hábitos deformantes y sexo

\begin{tabular}{|c|c|c|c|c|c|c|}
\hline \multirow{2}{*}{$\begin{array}{c}\text { Hábitos } \\
\text { deformantes }\end{array}$} & \multicolumn{2}{|c|}{ Femenino } & \multicolumn{2}{c|}{ Total } \\
\cline { 2 - 7 } & No. & $\%^{*}$ & No. & $\%^{*}$ & \multicolumn{2}{c|}{$\%^{* *}$} \\
\hline Succión digital & 9 & 69,2 & 4 & 30,8 & 13 & 22,8 \\
\hline $\begin{array}{c}\text { Uso del } \\
\text { biberón }\end{array}$ & 13 & 59,1 & 9 & 40,9 & 22 & 38,6 \\
\hline $\begin{array}{c}\text { Hábitos } \\
\text { posturales }\end{array}$ & 6 & 46,2 & 7 & 53,8 & 13 & 22,8 \\
\hline $\begin{array}{c}\text { Respiración } \\
\text { bucal }\end{array}$ & 10 & 43,5 & 13 & 56,5 & 23 & 40,4 \\
\hline
\end{tabular}

* \% calculado en base al total de casos por hábitos.

** \% calculado en base al total de niños. 
En la tabla 4 , se muestra un predominio de la dentición temporal presente en el $57,9 \%$ de los niños estudiados, a expensas de los que tenían 4 años de edad, mientras que en los restantes 24 niños (42,1\%), se observó la presencia de dentición mixta temprana, a expensas de los que tenían 5 años cumplidos.

Tabla 4. Niños de 4 a 6 años de edad con interferencias dentarias según edad y tipo de dentición

\begin{tabular}{|c|c|c|c|c|c|}
\hline \multirow{2}{*}{ Edad } & \multirow{2}{*}{ Casos } & \multicolumn{4}{|c|}{ Tipo de dentición } \\
\cline { 3 - 6 } & & \multicolumn{2}{|c|}{ Temporal } & \multicolumn{2}{c|}{ Mixta temprana } \\
\cline { 3 - 6 } & & No. & $\%^{*}$ & No. & $\%^{*}$ \\
\hline $\mathbf{4}$ & 25 & 22 & 88,0 & 3 & 12,0 \\
\hline $\mathbf{5}$ & 28 & 8 & 28,6 & 20 & 71,4 \\
\hline $\mathbf{6}$ & 4 & 3 & 75,0 & 1 & 25,0 \\
\hline Total & 57 & 33 & $57,9^{\star *}$ & 24 & $42,1^{\text {** }}$ \\
\hline
\end{tabular}

* \% calculado en base al total de casos por edades.

** \% calculado en base al total de niños.

La tabla 5 muestra la relación entre los hábitos bucales deformantes y la presencia de anomalías. Como habíamos visto anteriormente, el mayor número de niños no presentaron anomalías; sin embargo, el 38,5 \% ingiere leche en biberón y el 23,1
\% tiene disfunción respiratoria. En la mordida abierta anterior, el hábito que más estuvo presente fue la succión digital, seguido por la disfunción respiratoria y el uso del biberón para un 45,5\%.

Tabla 5. Niños de 4 a 6 años de edad con interferencias dentarias, según hábitos deformantes y presencia de anomalías

\begin{tabular}{|c|c|c|c|c|c|c|c|c|c|}
\hline \multirow{3}{*}{ Anomalías } & \multirow{3}{*}{$\begin{array}{c}\text { Total } \\
\text { de } \\
\text { casos }\end{array}$} & \multicolumn{8}{|c|}{ Hábitos deformantes } \\
\hline & & \multicolumn{2}{|c|}{ Succión digital } & \multicolumn{2}{|c|}{ Uso del biberón } & \multicolumn{2}{|c|}{$\begin{array}{c}\text { Hábitos } \\
\text { posturales }\end{array}$} & \multicolumn{2}{|c|}{$\begin{array}{l}\text { Disfunción } \\
\text { respiratoria }\end{array}$} \\
\hline & & No. & $\%$ & No. & $\%$ & No. & $\%$ & No. & $\%$ \\
\hline $\begin{array}{c}\text { Mordida } \\
\text { abierta } \\
\text { anterior }\end{array}$ & 11 & 7 & 63,6 & 5 & 45,5 & 2 & 18,2 & 6 & 54,5 \\
\hline $\begin{array}{l}\text { Oclusión } \\
\text { invertida } \\
\text { anterior }\end{array}$ & 4 & 0 & 0 & 1 & 25,0 & 2 & 50,0 & 2 & 50,0 \\
\hline $\begin{array}{l}\text { Oclusión } \\
\text { invertida } \\
\text { posterior } \\
\text { unilateral }\end{array}$ & 6 & 1 & 16,7 & 1 & 16,7 & 2 & 33,3 & 1 & 16,7 \\
\hline $\begin{array}{c}\text { Seudo clase II } \\
\text { funcional }\end{array}$ & 4 & 0 & 0 & 1 & 25,0 & 2 & 50,0 & 2 & 50,0 \\
\hline Sin anomalías & 26 & 3 & 11,5 & 10 & 38,5 & 2 & 7,7 & 6 & 23,1 \\
\hline
\end{tabular}

$\%$ en base al total de casos por fila

\section{DISCUSIÓN}

No hubo mayores limitaciones en este estudio, se trabajó con todos los niños que reunían las características y requisitos mencionados en el método.
Diversos estudios han demostrado que la edad no es un buen indicador para señalar cualquier suceso relacionado con el crecimiento y desarrollo del individuo, por su gran variabilidad, de ahí que sea tomada solo como un punto de referencia ${ }^{(13)}$.

Estos resultados difieren de los encontrados por Cabrera Sánchez ${ }^{(14)}$ en un estudio realizado en el 
círculo infantil Ana de Quesada de Santiago de Cuba, pues las interferencias dentarias se observaron casi por igual en el sexo masculino (25, para $35 \%$ ) que en el femenino (25, para $31,2 \%)$, aproximadamente el $30 \%$ en cada grupo.

Por otro lado , Vieira D. ${ }^{(15)}$ en un estudio realizado en niños encontró 39 niños con interferencias oclusales de los cuales, 25 (64,1\%) tenían 4 años de edad, número similar al nuestro en cuanto a ese grupo de edad, a diferencia de que no los divide por categoría bilógica. Otaño Lugo ${ }^{(16)}$ solo encontró 30 niños entre los 3 y 7 años de edad con interferencias dentarias de ambos sexos.

En investigación realizada con el propósito de conocer cómo se manifiestan las interferencias oclusales en la población infantil, Alemán Sánchez ${ }^{(17)}$ encontró 30 niños $(50,8 \%)$ que presentaban interferencias oclusales de relación céntrica a posición de máxima intercuspidación y en 29 (49,1\%) estas ocurrían durante los movimientos de lateralidad mandibular; estas cifras incluían ambos sexos entre los cuatro y cinco años de edad.

La lactancia materna en los primeros meses de vida produce la excitación muscular que favorece el crecimiento anteroposterior y transversal de los maxilares, sobre todo de la mandíbula, el desarrollo de los pterigoideos y la diferenciación de las articulaciones temporomandibulares; esto prepara al sistema muscular del niño para poder comer alimentos duros y secos, en cuanto erupción en sus dientes temporales y se establezca la oclusión dentaria ${ }^{(18)}$.

La Organización Mundial de la Salud (OMS) y el Fondo de Naciones Unidas para la Infancia (UNICEF) señalan que la lactancia «es una forma inigualable de facilitar el alimento ideal para el crecimiento y desarrollo correcto de los niños", recomendando como imprescindible la lactancia materna exclusiva durante los seis primeros meses del recién nacido. También recomiendan seguir amamantando a partir de los seis meses, al mismo tiempo que se va ofreciendo al bebé otros alimentos complementarios, hasta un mínimo de dos años ${ }^{(19)}$.

Estos resultados nos alertan sobre la necesidad de incrementar las acciones de promoción y prevención de salud en conjunto con el médico, la enfermera y los factores sociales para concientizar a las madres sobre la importancia de la lactancia materna.

El $5 \%$ más de niños menores de seis meses consumen fórmula y aumentó el porcentaje de niños que además de leche materna consumen innecesariamente agua. Esto es negativo porque inhibe la producción láctea e incrementa de manera importante el riesgo de enfermedades gastrointestinales ${ }^{(20)}$.

En el estudio de Cabrera Sánchez ${ }^{(14)}$, los resultados también difieren del nuestro, pues el $85,0 \%$ de los niños de su investigación tuvieron una lactancia no adecuada, de los cuales el 62,5\% tenían interferencias dentarias. Mientras que en investigación Cabrera Sánchez ${ }^{(14)}$ los resultados revelan que el $63.8 \%$ tuvieron una lactancia materna adecuada (exclusiva hasta los 6 meses) con predominio del sexo masculino.

Ferreira A. ${ }^{(12)}$ demostró que la presencia de hábitos bucales deformantes está más relacionado con el estado nutricional que presenten los niños, encontrando relevante la presencia de respiración bucal y el uso del biberón en niños con estados nutricionales desfavorables. Ruiseco A. ${ }^{(11)}$ encontró 148 niños respiradores bucales para el $24,6 \%$, con discreto predominio $(51,3 \%)$ del sexo femenino sobre el masculino, firmando que no existe predisposición genética en cuanto al sexo para la aparición de este hábito.

Se ha observado una alta incidencia relativa de la mordida cruzada posterior en los estadíos tempranos del desarrollo oclusal, en el caso de la dentición primaria y en la primera fase de la dentición mixta puede ser debida a causas dentarias, esqueléticas y funcionales. Esta última causa implica una adaptación funcional a las interferencias dentarias o en un intento de evitar las interferencias oclusales, causadas por la anchura inadecuada de la arcada dentaria, el niño realiza una adaptación funcional, desviando la mandíbula lateralmente, durante el cierre, para conseguir la intercuspidación ${ }^{(13)}$.

Los resultados pueden obedecer a que las malas posturas que adoptan los niños en la infancia durante el horario del sueño, de lectura y actividades diurnas pueden traer como resultado la aparición de alteraciones oclusales y deformidades faciales, en el caso de la succión digital existen algunos factores que la condicionan como: la falta de cariño, hambre, ansiedad o inducidos por los padres.

Cabrera Sánchez ${ }^{(20)}$, también obtuvo un predominio de niños con interferencias oclusales que se encontraban en el periodo de dentición temporal $(42,5 \%)$, el resto $(23,7 \%)$ se encontró en el periodo de dentición mixta. 
Se concluye que en los niños de 4 a 6 años de edad con interferencias dentarias de círculos infantiles predominó el sexo masculino y el periodo de dentición temporal. La respiración bucal y el uso del biberón fueron los hábitos deformantes más practicados, influenciados por una dieta y lactancia materna inadecuadas. Sin embargo la mayoría de los niños examinados no presentaron anomalías de la oclusión.

Contribuciones de autoría: BOHM, NLA, YPP, LBPH y NINA diseñaron el estudio, recopilaron y analizaron los datos, redactaron y aprobaron el manuscrito.

Fuente de financiamiento: Autofinanciado.

Conflicto de intereses: Los autores declararon no tener conflictos de interés.

\section{REFERENCIAS}

1. Ferreira A, Ramírez ML, García I, Ameneiros O Fernández $\mathrm{E}$. Tratamiento de interferencias oclusales en niños de 3 a 9 años de edad. Policlínico 19 de Abril [Tesis]. Habana: Universidad de la Habana. Escuela de Odontología. 2015.

2. Ruiseco A, Llanes M, Rodríguez OL, Rodríguez A. Dolor articular y su relación con Interferencias Oclusales. Revista Habanera de Ciencias de la Salud 2014; 13(3): 417-424.

3. Lazo Amador Y, Peñalver Soa S, Casamayor Laime Z. Necesidad de tratamiento ortodóncico en estudiantes de preuniversitario. Rev Cub Med Mil. 2014 [citado 7 Abr 2017]; 43(1). Disponible en:http://scielo.sld.cu/scielo.php? script=sci arttext\&pid=S013865572014000100002\&lng=es

4. García Peláez SY, Martín Zaldivar L, Lage Ugarte $M$, Altunaga Carbonel A. El mantenedor de espacio en la prevención de maloclusiones. AMC. 2014 [citado 10 Abr 2017]; 18(2). Disponible en: http://scielo.sld.cu/scielo.php?script=sci arttext\&pid=S1025-02552014000200005\&lng=es

5. León Alfonso J, Carvajal Roque J, Pérez Hermida $\mathrm{N}$, Rodríguez Martín $\mathrm{O}$. Hábitos bucales deformantes y su posible influencia sobre el plano poslácteo en niños con dentición temporal. MEDICIEGO. 2014 [citado 10 Abr 2017]; 20(2). Disponible

en:http://www.bvs.sld.cu/revistas/mciego/vol20_n 02_14/pdf/T8.pdf

6. Vergara Terrado RO, Barrueco Botiel LB, Díaz del Mazo L, Pérez Aguirre E, Sánchez Ochoa T. Influencia de la lactancia materna sobre la aparición de maloclusiones en escolares de 5 a 6 años. MEDISAN. 2014 [citado 10 Abr 2017]; 18(8). Disponible en: http://scielo.sld.cu/scielo.php?script=sci_arttext\& pid=S1029 $-30192014000800005 \& \operatorname{lng}=\mathrm{es}$

7. Cañete Vázquez RA, Frías Gutiérrez OA, Osoria Machado BE. Alteraciones en la oclusión de niños en edades de 3 a 5 años. Multimed. 2013; 17(2):140-52.

8. Reyes Romagosa DE, Torres Pérez I, Quesada Oliva LM, Milá Fernández M, Labrada Estrada HE. Hábitos bucales deformantes en niños de 5 a 11 años. MEDISAN. 2014 [citado 10 Abr 2017]; 18(5). Disponible en: http://scielo.sld.cu/scielo.php?script= sci_arttext\&pid=S102930192014000500003\&lng=es

9. . Díaz Méndez HT, Ochoa Fernández B, Paz Quiñones L, Casanova Sales K, Coca García Y. Prevalencia de maloclusiones en niños de la escuela Carlos Cuquejo del municipio Puerto Padre, Las Tunas. Medisur. 2015 [citado 7 Abr 2017]; 13(4). Disponible en: http://scielo.sld.cu/scielo.php?script=sci_arttext\& pid=S1727-897X2015000400005\&lng=es

10.Arocha Arzuaga A, Aranda Godínez MS, Pérez Pérez Y, Granados Hormigó AE. Maloclusiones y hábitos bucales deformantes en escolares con dentición mixta temprana. MEDISAN. 2016 [citado 7 Abr 2017]; 20(4). Disponible en:http://scielo.sld.cu/scielo.php?script=sci_arttex t\&pid=S1029-30192016000400002\&Ing=es

11.Ruiseco A, Llanes M, Rodríguez OL, Rodríguez A. Dolor articular y su relación con Interferencias Oclusales. Revista Habanera de Ciencias de la Salud 2014; 13(3): 417-424.

12.Ferreira A, Ramírez ML, García I, Ameneiros O Fernández $E$. Tratamiento de interferencias oclusales en niños de 3 a 9 años de edad. Policlínico 19 de Abril [Tesis]. Habana: Universidad de la Habana. Escuela de Odontología. 2015.

13.Pérez Varela $\mathrm{H}$, Ramos Morales $\mathrm{C}$, Domínguez Fleites LM. Tratamiento precoz de interferencias oclusales que provocan laterognatismo en niños de edades tempranas. Rev. Cubana Ortod. 1988 [consultado: 30 agosto 2016]; 13(2) Disponible en: http://www.bvs.sld.cu/revistas/ord/vol13298/ord0 3298.htm

14.Cabrera Sánchez TV, George Valls Y, Martínez Ramos MR, Ramírez Quevedo Y, González Esplanger L. Estado de la oclusión y tratamiento selectivo en niños con dentición temporal y mixta temprana. Rev. Cubana Estomatol. 2016 [consultado 16 abril 2017]; 20(3) Disponible en: http://scielo.sld.cu/scielo.php?script=sciarttext\&pi $\mathrm{d}=$ S0034-7507200000200004 
15. Vieira D. Características esenciales de la oclusión temporal ideal. Propdental. 2014 [citado 25 Dic 2016]; 18(2):1-5.

16. Otaño Lugo R, Massón Barceló RM, Fernández Ysla R, Llanes Rodríguez M, Cruz Rivas $\mathrm{Y}$, Delgado Carrera L, et al. Ortodoncia. La Habana: Editorial Ciencias Médicas; 2014.17. Sonis S. Dental secrets. 4 ed. Cambridge: Elsevier; 2015.

17. Alemán Sánchez $P$, González Valdés $D$, Concepción Acosta RB. Anomalías dentomaxilofaciales y sus factores de riesgo. Rev Haban Cienc Méd. 2015 [citado 25 Dic 2016]; 14(2): 179-87.

18. Montero Parrilla JM, Morais Chipombela LC, Semykina O. La oclusión dentaria en interacción con la postura corporal. Rev Cubana Estomatol. 2014 [citado 24 Dic 2016] ; 51(1): 15-23.
19. Bilgic, F.; Gelgor, I. E. \& Celebi, A. A. Malocclusion prevalence and orthodontic treatment need in central Anatolian adolescents compared to European and other nations' adolescents. Dental Press J. Orthod., 20(6):75-81, 2015.

20. Cabrera Sánchez TV, Martínez Ramos MR, Comas Mirabent R, González Esplanger L, Perú Seguí $Y$. Interferencias oclusales en niños con dentición temporal y mixta temprana. MEDISAN. Santiago de Cuba, marzo 2015 [Consultado: 30 agosto 2016]; 19(3) Disponible en: http://scielo.sld.cu/scielo.php?script=sciarttext\&pi d=S1029-0192015000300005

\author{
Bárbara Olaydis Hechavarría Martínez \\ barbaraolaidis@infomed.sld.cu \\ Leonardo Núñez Antúnez \\ edgardo.nunez@infomed.sld.cu \\ Yisel Pérez Pérez \\ yisel.perez@infomed.sld.cu \\ Leanne Bárbara Pons Hechavarría \\ lia.baby@nauta.cu \\ Nayra Ivonne Núñez Almarales \\ nivonne@nauta.cu
}

(D) orcid.org/0000-0002-8570-6947

(D) orcid.org/0000-0002-2516-9828

orcid.org/0000-0001-6117-3448

orcid.org/0000-0003-4753-7633

orcid.org/0000-0002-2713-7117 